\title{
Watermarked 3-D Mesh Quality Assessment
}

\author{
Massimiliano Corsini, Elisa Drelie Gelasca, Touradj Ebrahimi, Member, IEEE, and \\ Mauro Barni, Senior Member, IEEE
}

\begin{abstract}
This paper addresses the problem of assessing distortions produced by watermarking 3-D meshes. In particular, a new methodology for subjective evaluation of the quality of 3-D objects is proposed and implemented. Two objective metrics derived from measures of surface roughness are then proposed and their efficiency to predict the perceptual impact of 3-D watermarking is assessed and compared with the state of the art. Results obtained show good correlations between the proposed objective metrics and subjective assessments by human observers.
\end{abstract}

Index Terms-3-D objects quality assessment, 3-D watermarking, mesh watermarking, objective metrics, perceptual metrics, subjective evaluation.

\section{INTRODUCTION}

$\mathbf{I}_{\mathrm{a}}^{\mathrm{N}}$ $\mathrm{N}$ the last decade, digital watermarking has become a very active research topic with important applications in the fields of copyright protection for multimedia content, digital rights management, document authentication and conditional access to enhanced services. Until a few years ago, research efforts have been mainly focused on the watermarking of audio, image and video data; only recently has watermarking of 3-D objects gained attention, due to the ever-increasing diffusion of such objects in many areas including architecture, design, mechanical engineering, cultural heritage, and entertainment. Hence, watermarking of 3-D objects has not reached the same level of maturity as for still images and video.

One of the main requirements of any watermarking algorithm is the imperceptibility of the watermark. This is also a fundamental constraint for 3-D watermarking. It is then very important that suitable methodologies are developed to measure the quality of the watermarked objects as judged by human observers. Generally speaking, there are two major classes of quality criteria evaluations: objective and subjective. In fact, one obvious way of determining the quality of any visual information is to measure it by means of subjective experiments

Manuscript received January 3, 2006; revised January 31, 2006. This work was supported in part by the Italian Ministry of Research and Education under FIRB Project RBIN04AC9W. The associate editor coordinating the review of this paper and approving it for publication was Dr. Stefanos Kollias.

M. Corsini is with Visual Computing Laboratory, Istituto di Scienza e Tecnologia dell'Informazione, National Research Council, Pisa 56124, Italy (e-mail: massimiliano.corsini@isti.cnr.it).

E. D. Gelasca is with Vision Research Laboratory, Department of Electrical and Computer Engineering, University of California, Santa Barbara, CA 93106 USA (e-mail: elisa.drelie@a3.epfl.ch).

T. Ebrahimi is with the Signal Processing Institute, Ecole Polytechnique Fédérale de Lausanne (EPFL), Lausanne, Switzerland (e-mail: touradj.ebrahimi@epfl.ch).

M. Barni is with the Department of Information Engineering, University of Siena, 53100 Siena, Italy (e-mail: barni@dii.unisi.it).

Color versions of Figs. 1 and 5 are available online at http://ieeexplore.ieee. org.

Digital Object Identifier 10.1109/TMM.2006.886261 with human observers. However, such subjective evaluations are not only time-consuming and expensive, but they also cannot be incorporated into automatic systems. The goal of this paper is to develop objective measures that can automatically predict the quality of a watermarked 3-D mesh as perceived by human subjects. Due to the novelty of quality evaluation of 3-D data, no standardized procedures exist and current studies show this lack of generalization [1]. Nevertheless, since subjective quality measures exhibit some inherent drawbacks (the use of a standard evaluation room, a large panel of human observers, etc.), there has been a great interest in developing objective metrics for 3-D model quality assessment. In this work, we propose two perceptual metrics for the quality assessment of watermarked 3-D objects. Note that, admittedly, our new metric will not represent a general tool to evaluate the quality of a 3-D model, but it will only serve the purpose of judging the quality of watermarked meshes. The final aim of our study is to provide a mean whereby watermarking researchers can evaluate the degradation introduced by the watermark and take appropriate countermeasures to minimize it. At the same time, the proposed metric will be particularly useful for comparing the performance of different 3-D watermarking algorithms.

A possible approach could be to simply apply the perceptual metrics developed for still images to the final rendered views of the 3-D model. The main problem of this approach is that the perceived degradation of still images may not be adequate to evaluate the perceived degradation of the equivalent 3-D model [1]. Hence, the approach we chose is to evaluate the human perception of geometric distortions in watermarked models and then to build ad-hoc perceptual metrics that work directly on the model's surface. In such a case, subjective experiments dealing directly with the 3-D models are needed.

The paper is organized as follows. The state-of-the-art related to the research addressed in this work is reviewed in Section II. The experimental methodology is described in Section III. In Section IV we describe the subjective experiments and the artifacts introduced by common 3-D watermarking algorithms. Section V describes the proposed metrics. Finally, results are presented and discussed in Section VI before drawing the conclusions in Section VII.

\section{RELATED WORK}

Several methods have been proposed so far to exploit the models of the Human visual system (HVS) to improve the effectiveness of existing watermarking schemes [2], [3]. We can divide the approaches proposed so far into theoretical [3], [4] and heuristic [5], [6].

Concerning quality evaluation, in the past years, a set of techniques have been defined and proposed both subjectively [7], [8], and objectively [9] to be used for watermarked video quality 
evaluation. To the best of our knowledge no similar standards have been proposed for watermarked 3-D meshes. In recent years, in order to properly evaluate mesh simplification and perceptually guided rendering of 3-D objects, few objective metrics have been proposed, but even less attention has been paid to establishing a reliable procedure for subjective evaluation of 3-D data and results show the complexity of such a task [1].

Mesh simplification reduces the number of vertices and triangles of a polygonal mesh model while preserving its visual appearance. In general, the simplification process is driven by a similarity metric that measures the impact of the changes of the model after each simplification step. Two kinds of metrics are usually adopted: geometric metrics and (perceptual) imagebased metrics. The most used global geometry-based metrics for off-line quality evaluation of 3-D models are the maximum geometric error and mean geometric error based on the Hausdorff distance [10], [11]. Concerning perceptual image-based simplification, Lindstrom and Turk [12] propose an image-driven approach for guiding the simplification process: the model to be simplified is rendered by considering several viewpoints and an image quality metric is used to evaluate the perceptual impact of the simplification operation. More recently, Williams et al. [13] developed a view-dependent simplification algorithm based on a simple model of the contrast sensitivity function that takes into account texture and lighting effects.

The aim of perceptually guided rendering is to accelerate photo-realistic rendering algorithms to avoid computations that do not impact the perceived final result. Some remarkable works in this field include Bolin and Meyer [14], and the work by Ferwarda et al. [15], where a sophisticated perceptual metric for the evaluation of how much a visual pattern, i.e., a texture, hides geometry artifacts is proposed. The visual masking effect caused by texturing is taken into account by analyzing the final rendered images.

As we said, a possibility could be to apply image-based perceptual metrics to the final rendered images of the 3-D model. This approach was investigated by Lindstrom and Turk [12]. The main problem is that the perceived degradation of still images may not be adequate to evaluate the perceived degradation of the equivalent 3-D model as concluded by the subjective experiments by Rogowitz and Rushmeier [1]. Another possible approach is to evaluate how the human visual system perceives geometric distortions on the model surface and to build an ad-hoc perceptual metric for geometric artifacts. The latter approach is more interesting from a research viewpoint, since no similar studies have been conducted so far. The potential field of applications is not limited to 3-D watermarking, but other computer graphics applications can also benefit from them. For these reasons, this work has adopted the second approach, i.e., to work directly on the geometry of the 3-D model.

\section{A Methodology for SubJective Quality Evaluation}

Since no standards exist for the evaluation of the quality of 3-D objects with impairments, we propose a method for subjective evaluation of watermarked 3-D objects based on the criteria usually followed in video and multimedia content quality evaluation [7], [8].
In designing subjective experiments for quality evaluation of 3-D objects, a first crucial problem is to decide the way the object under examination is rendered. This is not a trivial task. Hence, an accurate study has been carried out. For instance, the rendering conditions should not bias the human perception of the 3-D model by privileging, for example, one view of the 3-D object rather than another. In our investigation, the rendering conditions have been set as follows.

- Nonuniform background. The 3-D model is visualized on a nonuniform background in order to not overestimate the importance of the contour of the model with respect to its overall shape. The background color fades from blue (at the top) to white (at the bottom).

- Light source. All models are illuminated with a single white point light source since multiple lights can confuse the observer [16]. To be more specific, each model is illuminated with one white point light source located in a top corner of the object bounding box (OBB) of the 3-D object.

- Lighting. We use a simple local illumination lighting model where only the diffusive component of the reflected light is considered to avoid the dependence on camera's position.

- Texturing. Image texture mapping, bump mapping, and other kinds of texturing usually produce masking effects on the perceived geometry [15] and consequently on the perception of watermarking artifacts. Since we leave visual masking studies specific for 3-D models as a future research area we do not account for such visual effects in our metrics. For theoretical considerations about visual masking and texturing, we refer to Section VI-D

- Material properties. The color of a surface is determined by the parameters of the light source that illuminate the surface, by the lighting model used and by the properties of the surface's material. We set the material properties to obtain a stone-like effect. This choice is made for different reasons: first, if all models are seen as "statues" the subjects perceive them in a natural way; second, in this way, the memory color phenomenon is avoided [17]. The memory color phenomenon describes the fact that an object's characteristic color influences the human perception of that object's color, e.g., shapes such as heart and strawberries are characteristically red.

- Screen and Models Resolution. The monitor resolution used in the experiments is $1280 \times 600$ and each model is displayed in a $600 \times 600$ window. The model occupies about $80 \%$ of such window and the resolution of the models ranges between 50000 and 100000 triangles allowing for a good visualization of the details.

- Interaction. The studies of Rogowitz and Rushmeier [1] suggest that an experimental methodology to evaluate the perceived alterations of 3-D objects should rely on the interaction with the model. For this reason, we decided to allow the subject to interact with the model by rotating and zooming it.

Eleven test subjects (one female, ten males) were drawn from a pool of students aged between 24 and 30. The 3-D models were displayed on an uncalibrated 17-in LCD monitor, with participants sitting approximately $0.4 \mathrm{~m}$ from the display. The experi- 
ments followed a five-stage procedure. The stages were 1) oral instructions; 2) training; 3) practice trials; 4) experimental trials; and 5) interview. In the first stage, the subjects were verbally instructed and made familiar with the task and the graphic interface. In the training, the original models and the watermarked models were shown to establish the range for the impairment scale. The practice trials stage was used to familiarize subjects with the experimentation. In the experimental stage, the subjects had to give a score to indicate how much the distortions were evident. The subjects were instructed to enter a numerical value greater than 0 proportional to the distortion noticed. The value of " 10 " had to be assigned to the most evident distortion representing the worst cases shown in the training phase. Finally, in the interview stage, test subjects were asked to provide a qualitative description of the perceived artifacts.

\section{SUBJECTIVE EXPERIMENTS}

Two sets of subjective experiments were carried out with different purposes. The first set of experiments (Experiment I), were performed to tune the two objective metrics (proposed in Section V) with psychovisual data in order to transform them into two perceptual metrics.

In this first set of experiments, test subjects evaluated differently watermarked models ranging from severe down to weak visual impairments. Those different distortion strengths were generated using a specific watermarking algorithm, i.e., the algorithm by Uccheddu et al. (UCB) [18]. The second set of experiments, (Experiment II) were conducted to validate the proposed metrics. Specifically, in Experiment II, we implemented and adopted three other different watermarking algorithms: the vertex flood algorithm (VFA) [19], the normal bin encoding (NBE) [20], and the method by Kanai et al. (KDK) [21]. Concerning the kind of impairments introduced in the model, the UCB algorithm produced a uniform noise that can be described as an increase of the roughness of the watermarked surface. VFA produced a noise similar to a marble streak. The artifacts of the KDK algorithm were similar to those obtained by the UCB algorithm but due to the geometric tolerance introduced by Kanai to limit the visual impact of the watermarking, the final visual effects of such distortions were not uniformly distributed over the model surface. Concerning NBE, the visual aspect (crack-like) of its artifacts was different from that of UCB, VFA, and KDK and more difficult to perceive. Fig. 1 shows a detail of the model "Horse" after application of the four different watermarking algorithms.

The test models used for both the experiments were: "Bunny", "Horse", "Venus", and "Feline". A total of 40 (4 originals $\times 3$ watermarking strength $\times 3$ resolution level +4 originals) test models were used in Experiment I. A total of 48 ( 4 models $\times 11$ watermarking settings +4 originals) test models were used in Experiment II.

\section{Proposed Perceptual Metrics}

Thanks to the intuition and to previous studies about visual aspects of 3-D watermarking [22], we argued that a good measure of the visual artifacts produced by watermarking should be based on the amount of roughness introduced on the surface. Moreover, the interviews in Experiments I and II confirmed that the different types of noise on the surfaces produced by the watermarking can be described essentially with the term roughness. Hence, we chose to measure the strength of the artifacts on the basis of an estimation of the surface roughness. In particular, two objective metrics based on roughness estimation of the surface have been developed.

\section{A. Multiscale Roughness Estimation}

The first roughness measure is a variant of the method by Wu et al. [23]. This metric measures the per-face roughness by making statistical considerations about the dihedral angles associated to each face. Wu et al. developed this measure in order to preserve significant shape features in mesh simplification algorithms.

The dihedral angle is the angle between two planes. For a polygonal mesh, the dihedral angle is the angle between the normals of two adjacent faces [Fig. 2(a)]. The basic idea of this method [24] is that the dihedral angle is related to the surface roughness. In fact, the face normals of a smooth surface vary slowly over the surface, consequently the dihedral angles between adjacent faces are close to zero. To be more specific, $\mathrm{Wu}$ et al. associated to each dihedral angle an amount of roughness given by the quantity

$$
\rho_{d}=1-\left(\vec{N}_{1} \cdot \vec{N}_{2}\right)
$$

where - denotes the scalar product between two vectors, and the subscript $d$ was added to explicitly indicate that $\rho_{d}$ measures the roughness of a dihedral angle.

Given a triangle $T$ with vertices $v_{1}, v_{2}$ and $v_{3}$, its roughness is computed as

$$
\rho_{1}(T)=\frac{G\left(v_{1}\right) V\left(v_{1}\right)+G\left(v_{2}\right) V\left(v_{2}\right)+G\left(v_{3}\right) V\left(v_{3}\right)}{V\left(v_{1}\right)+V\left(v_{2}\right)+V\left(v_{3}\right)} .
$$

By referring to Fig. 2(b), $G\left(v_{1}\right)$ is the mean of the roughness associated to the dihedral angles $T-T_{1}, T_{1}-T_{2}, T_{2}-T_{3}$, $T_{3}-T_{4}, T_{4}-T_{5}$, and $T_{5}-T$. In the same way, $G\left(v_{2}\right)$ and $G\left(v_{3}\right)$ are the mean roughness associated to the dihedral angles of the faces adjacent to the vertices $v_{2}$ and $v_{3}$. Instead, $V\left(v_{1}\right)$, $V\left(v_{2}\right)$ and $V\left(v_{3}\right)$ are the variances of the roughness $\rho_{d}$ of the dihedral angles of the faces adjacent to the vertices $v_{1}, v_{2}$, and $v_{3}$.

A rough surface can be considered as a surface with a high concentration of bumps of different sizes. The roughness measure expressed in (2) is able to measure the 'bumpiness' of surfaces at the face level, but, if the granularity of the surface roughness, i.e., the size of the bump, is bigger than the average size of one face, this metric fails to measure it correctly. In other words, this measure does not take into account the scale of the roughness. Our idea was to modify equation (2) to account for different bump scales. The first step to achieve this goal is to transform the per-face roughness estimation in a per-vertex roughness in the following way:

$$
\rho_{1}^{N}(v)=\frac{1}{\left|S_{T}^{N}\right|} \sum_{i \in S_{T}^{N}} \rho_{1}\left(T_{i}\right) \mathcal{A}_{T_{i}}
$$




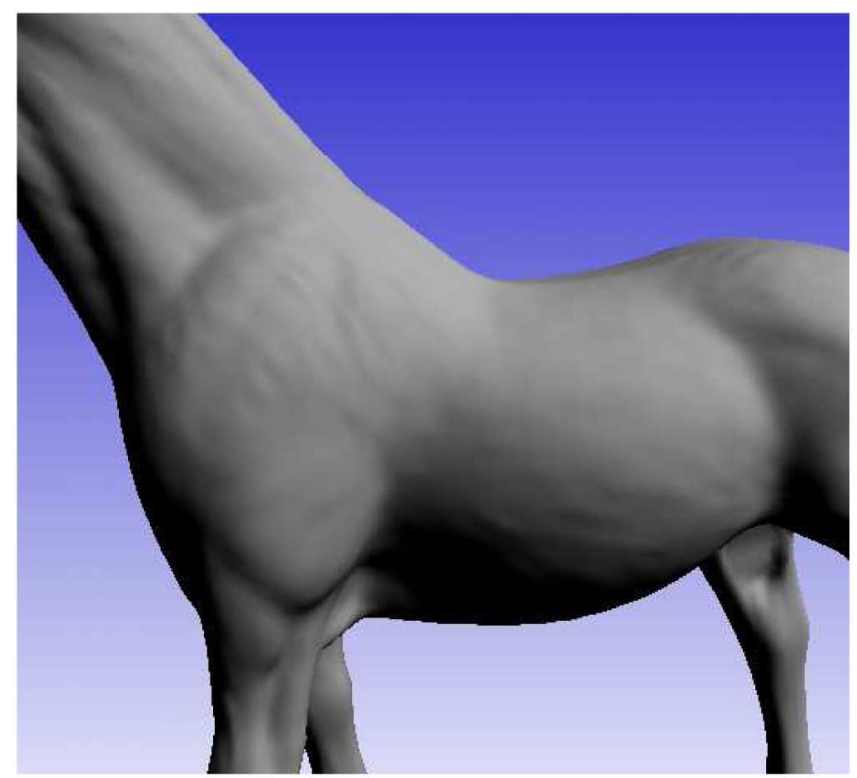

UCB algorithm

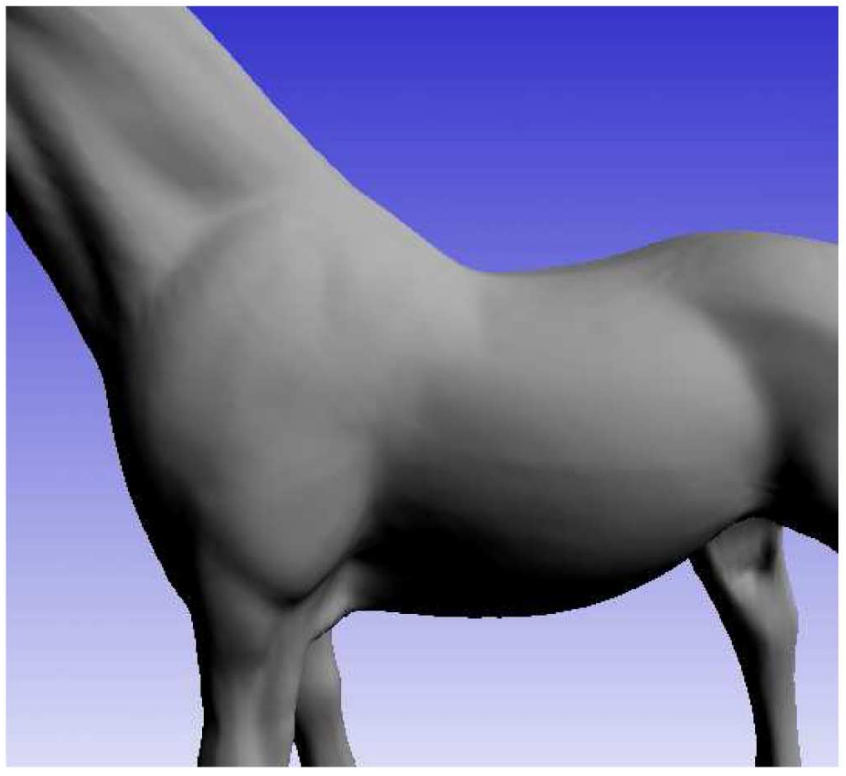

Normal Bin Encoding (NBE)

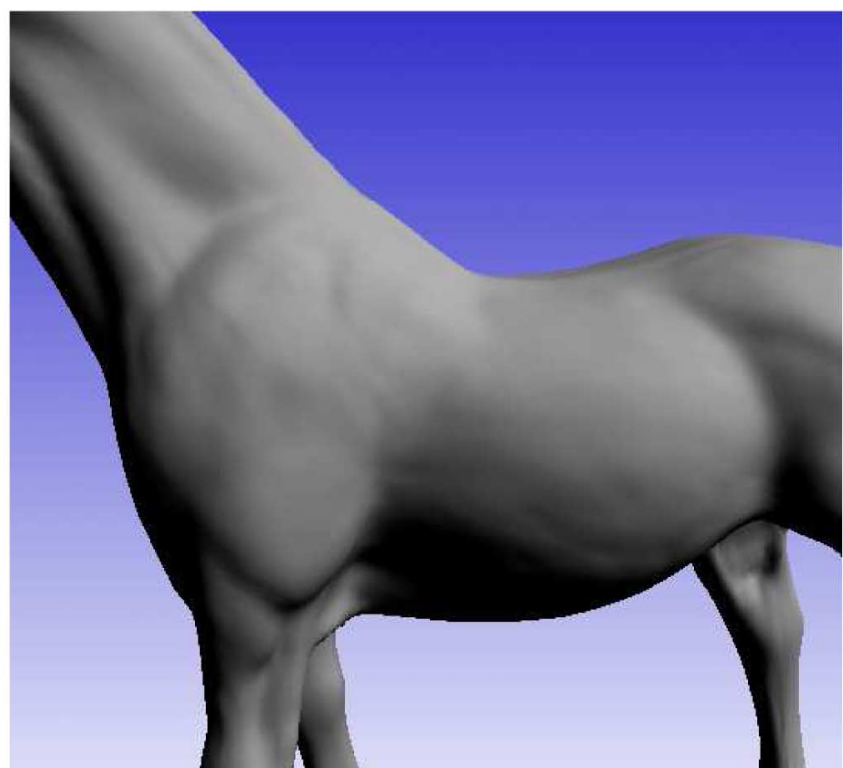

KDK algorithm

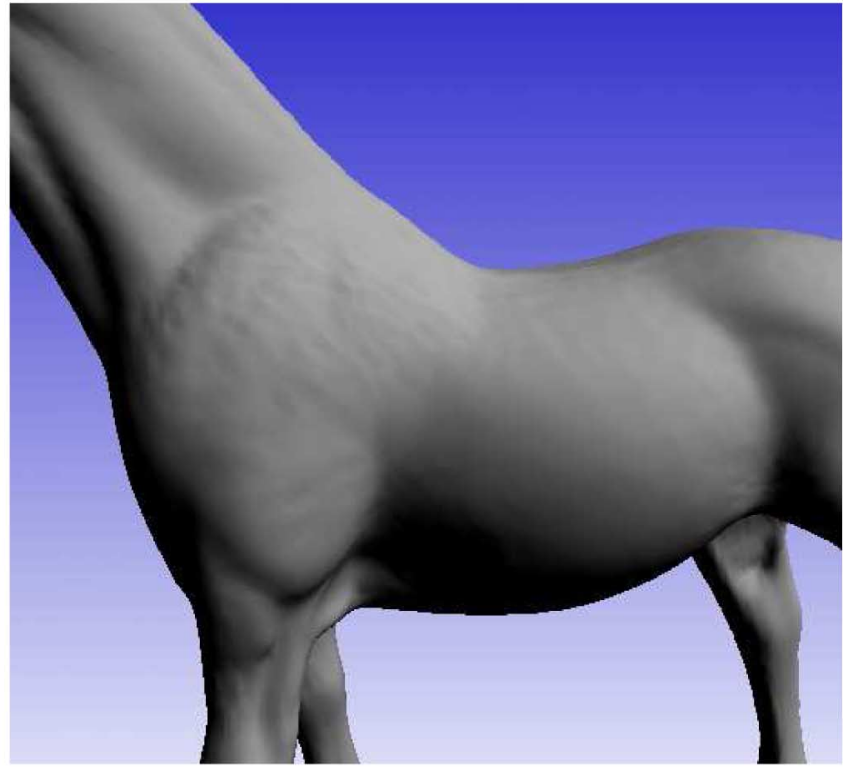

Vertex Flood Algorithm (VFA)

Fig. 1. Geometric defects introduced by 3-D watermarking algorithms.

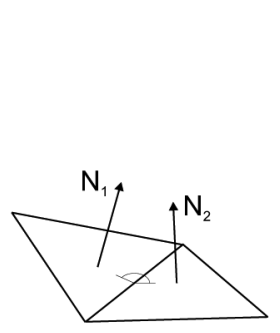

(a)

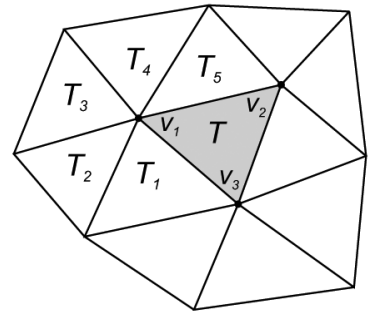

(b)

Fig. 2. (a) Dihedral angle. (b) Mean roughness $G(\cdot)$, and variance roughness $V(\cdot)$.

where $S_{T}^{N}$ is the set of the faces of the $N$-ring of the vertex $v$, $|\cdot|$ is the cardinality operator and $\mathcal{A}_{T_{i}}$ is the area of the face $T_{i}$. Considering the $\mathrm{N}$-ring in the roughness evaluation accounts for

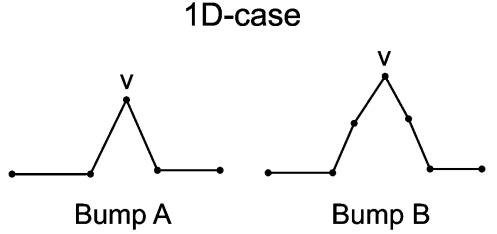

Fig. 3. Bumps with different scale.

different scales of bumpiness. By referring to Fig. 3, the bump of size equivalent to the 1 -ring (A) is well measured by $\rho_{1}^{1}(v)$, a correct value of roughness for the vertex $v$ in the case (B) is provided by $\rho_{1}^{2}(v)$. Approximately, we can state that the roughness of a vertex $v$ centered on a bump whose area is close to the area of the faces that form the N-ring is well measured by 


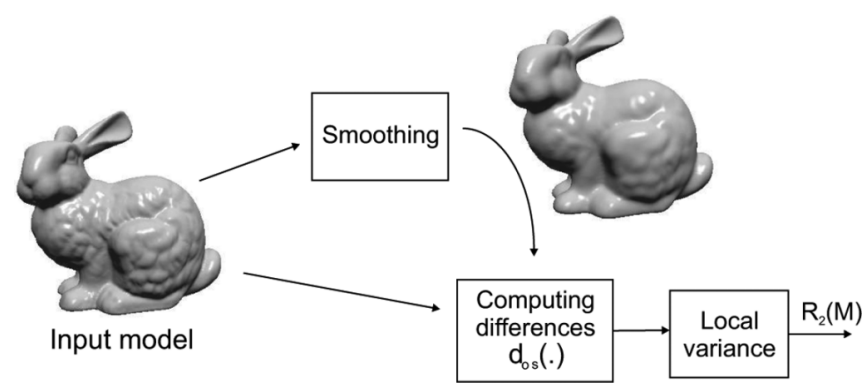

Fig. 4. Smoothing-based roughness estimation.

$\rho_{1}^{N}(v)$. This approximation could still create invalid estimates in certain cases. For example for high values of $N$, or when a surface presents high curvatures. Despite possible impairments, the proposed metric provides acceptable results in most cases. In order to obtain a single value of roughness that accounts for the roughness evaluated at several scales, the maximum value produced by N-rings of different sizes is retained. In our objective metric, after several tests, three scales of roughness: the 1-ring, the 2-ring, and the 4-ring were retained. Hence, the final per-vertex roughness metric becomes

$$
\rho_{1}(v)=\max \left\{\rho_{1}^{1}(v), \rho_{1}^{2}(v), \rho_{1}^{4}(v)\right\} .
$$

A metric using a linear scale of roughness, i.e., the 1-ring, the 2-ring, and the 3-ring, provides similar results.

The total roughness of the 3-D object is the sum of the roughnesses of all vertices

$$
\rho_{1}(M)=\sum_{i=1}^{N_{v}} \rho_{1}\left(v_{i}\right)
$$

where $N_{v}$ is the total number of mesh vertices. In the following, we describe how to transform this multiscale roughness estimation into an objective metric.

\section{B. Smoothing-Based Roughness Estimation}

The second method [25] is based on the consideration that artifacts are better perceived on smooth surfaces. This was confirmed by interviews with subjects. Hence, a smoothing-based roughness estimation was developed. The basic idea of this approach is to apply a smoothing algorithm to the model and then to measure the roughness of the surface as the variance of the differences between the smoothed version of the model and the original. A sketch of the smoothing-based roughness estimation process is given in Fig. 4.

The first step is to build a smoothed version of the model $\left(M^{S}\right)$ by applying a smoothing algorithm to the input model $(M)$. Several possibilities for smoothing exist [26]-[28]. Here, we decided to use the Taubin filter [26] for its simplicity of implementation. For the Taubin filter, we used the parameters $\lambda=0.6307, \mu=-0.6352$ that give a strong smoothing effect. The filter is iterated five times. Once the smoothed model is obtained, the distance between each vertex of $M$ and $M^{S}$ is computed in the following way:

$$
d_{O S}\left(v, v^{S}\right)=\operatorname{proj}_{\vec{n}_{v}^{S}}\left(v-v^{S}\right)
$$

where $\operatorname{proj}(\cdot)$ indicates the projection of the vector $\left(v-v^{S}\right)$ on the vertex normals of the smoothed surface $\left(\vec{n}_{v}^{S}\right)$. The projection along normals is used to evaluate the vertex-vertex distance since after the smoothing operation the vertices can move slightly from their original position. Hence, the simple Euclidean distance between corresponding vertices is not meaningful for our purpose. At this point the per-vertex roughness is computed by evaluating the local variance of the distances $d_{O S}(\cdot)$ around each vertex. To be more specific, for each vertex $v$, the set of distances associated to its 2-ring $\left(S_{d}^{2}(v)\right)$ is built and the variance of this set evaluated. Then, the per-vertex smoothing-based roughness is computed by

$$
\rho_{2}(v)=\frac{V\left(S_{d}^{2}(v)\right)}{\mathcal{A}_{S^{2}}}
$$

where $A_{S^{2}}$ is the area of the faces that form the 2-ring of $v$. This area is used as the denominator since surfaces with the same local variance of the distances but smaller area are assumed to be rougher. The roughness of the input model is the sum of the roughnesses of all the vertices

$$
\rho_{2}(M)=\sum_{i=1}^{N_{v}} \rho_{2}\left(v_{i}\right)
$$

where $N_{v}$ is the number of vertices of the model.

\section{Objective Metrics}

Now, we describe how to use the roughness estimation to predict the visual distortion produced by a certain 3-D watermarking algorithm. On the basis of several evaluations, we decided to define our objective metric as the increment of roughness between the original and the watermarked model. This increment is normalized with respect to the roughness of the original model, leading to

$$
\mathcal{R}\left(M, M^{w}\right)=\log \left(\frac{\rho\left(M^{w}\right)-\rho(M)}{\rho(M)}+k\right)-\log (k)
$$

where $\rho(M)$ is the total roughness of the original model and $\rho\left(M^{w}\right)$ is the total roughness of the watermarked model. Both $\rho_{1}(\cdot)$ and $\rho_{2}(\cdot)$ can be used to obtain two different objective metrics. Equation (9) is a sort of "Weber Law" in which the amount of roughness substitutes the grey levels. The logarithm is employed to better discriminate small values of relative roughness increments. The constant $k$ is used to avoid numerical instability since the logarithm tends to $-\infty$ for $\rho\left(M^{w}\right)$ very close to $\rho(M)$. Since, in the experiments, the subjects used values between 0 and 10, the value $k$ has been set to normalize the metric's outputs to the same range. Finally, it is important to underline that this metric is not influenced by vertex density since the normalized differences of model roughness are used. In the following, we will indicate with $\mathcal{R}_{1}\left(M, M^{w}\right)$ the objective metric based on the multi-scale roughness estimation and with $\mathcal{R}_{2}\left(M, M^{w}\right)$ the objective metric relying on the smoothing-based roughness estimation. 


\section{From Objective to Perceptual Metric}

Basically, there are two approaches [29] to model psychophysical quantities: performance modeling and mechanistic modeling. Although the distinction is more a continuum than a strict dichotomy, the performance models tend to treat the entire visual system as a "black box" for which input/output functions need to be specified. For the mechanistic model, physiological and psychophysical data are used to open the black box. For our metrics, we opted for the black box approach since a model of visual perception of geometric artifacts could become too complex to be handled in practice. In addition the application-oriented nature of our metrics makes the black box approach to be a more appropriate solution.

Before using the results of Experiment I to obtain our perceptual metric, we summarized the subjective scores.

The subjective scores have to be condensed by statistical techniques used in standard methods [8] to yield results which summarize the performance of the system under test. The averaged score values, mean opinion score (MOS), are considered as the amount of distortions that anyone can perceive on a particular watermarked 3-D object. However, impairment is measured according to a certain scale, and such a scale may vary from person to person. For this reason, we used standard methods to normalize and to screen the judgments provided by the subjects [7]. The MOS values obtained following the above approach were used to derive a perceptual metric by fitting them with a psychometric curve.

The purpose of a psychometric curve is to associate the values given by the objective metric to the subjective scores provided by the subjects. This step is always necessary in order to take into account the saturation effects typical of human senses. For these reasons, psychometric curves exhibit a typical sigmoid shape that penalizes the strongest stimuli. Through psychometric mapping, a match between the human perception of geometric artifacts and the values provided by the objective metric is established. In particular, we used the Gaussian psychometric function

$$
g(a, b, \mathcal{R})=\frac{1}{2 \pi} \int_{a+b \mathcal{R}}^{\infty} e^{-\left(t^{2} / 2\right)} d t
$$

where $a$ and $b$ are the parameters to be estimated by fitting the objective metric values to the subjective data, and $\mathcal{R}$ is the objective metric used to measure the visual distortions. We opted for this psychometric curve since it provided the best fit for our data among the commonly used curves, i.e., Gaussian, logistic, and Weibull curves [30]. To estimate the parameters $a$ and $b$, we used a nonlinear least-squares data fitting by the Gauss-Newton method.

\section{EXPERIMENTAL RESULTS}

In this section, we analyze the performance of the two proposed objective metrics and compare them with the geometric metrics usually adopted in the literature.

Evaluations are performed by assessing the correlations with the MOS described previously. The correlations between the subjective MOS collected in Experiment I and the distances
TABLE I

Perceptual Metrics Performances

\begin{tabular}{|c|c|c|c|c|c|c|}
\hline & \multicolumn{2}{|c|}{ Hausdorff Distance } & \multicolumn{2}{|c|}{$\mathcal{R}_{1}^{*}\left(M, M^{\mathbf{w}}\right)$} & \multicolumn{2}{|c|}{$\mathcal{R}_{2}^{*}\left(M, M^{\boldsymbol{w}}\right)$} \\
\hline Algo. & $\operatorname{Max}\left(r_{S}\right)$ & Mean $\left(r_{S}\right)$ & $r_{P}$ & $r_{S}$ & $r_{P}$ & $r_{S}$ \\
\hline UCB & 0.67 & 0.66 & 0.67 & 0.87 & 0.83 & 0.90 \\
\hline NBE & 0.71 & 0.70 & 0.56 & 0.80 & 0.62 & 0.81 \\
\hline VFA & 0.50 & 0.88 & 0.75 & 0.94 & 0.78 & 0.91 \\
\hline KDK & 0.70 & 0.32 & 0.62 & 0.72 & 0.55 & 0.71 \\
\hline $\begin{array}{l}\text { KDK } \\
\text { NBE } \\
\text { VFA }\end{array}$ & 0.38 & 0.49 & 0.49 & 0.71 & 0.50 & 0.69 \\
\hline $\begin{array}{l}\text { UCB } \\
\text { NBE } \\
\text { VFA }\end{array}$ & 0.52 & 0.62 & 0.65 & 0.84 & 0.74 & 0.90 \\
\hline
\end{tabular}

given by two geometric metrics based on the Hausdorff distance for model similarity are evaluated. The data of the Experiment II are used to validate the developed metrics on different watermarking algorithms.

First, the performance of the state of the art metrics currently used to evaluate the differences between 3-D models are analyzed. These state-of-the-art metrics are mean and maximum geometric error based on Hausdorff distance [10], [11]. Second, the performance of the proposed metrics are assessed and compared to those of the state-of-the-art metrics.

\section{A. Hausdorff Distances}

As previously stated (Section II), two of the most common geometric metrics, $d_{\infty}(\cdot)$ and $d_{1}(\cdot)$, are based on the Hausdorff distance between surfaces. Here, we want to evaluate if the Hausdorff distance between the original and the watermarked models could be a reliable metric for the prediction of the perceptual impairments introduced by the watermarking process. To do so, the Hausdorff distances between watermarked models and the originals are plotted versus the MOS provided by Experiment I. At this point, the linear correlation coefficient of Pearson $\left(r_{P}\right)$ [31] or the nonlinear (rank) correlation coefficient of Spearman $\left(r_{S}\right)$ [32] are calculated in order to evaluate the global performance of the geometric and proposed metrics obtained by fitting these geometric data with the cumulative Gaussian in (10). The geometric metric results do not correlate well with subjective $M O S$. This underlines the fact that $d_{\infty}(\cdot)$ and $d_{1}(\cdot)$ do not reflect how humans perceive geometric artifacts due to watermarking. The results are summarized in the first two columns of Table I, and will be used as a reference to compare with the performance of our perceptual metrics.

\section{B. Roughness-Based Metrics in Experiment I}

As stated before, the goal of the first experiment was to make an initial study on the perception of the geometric artifacts caused by watermarking algorithms and to lay the basis for the development of perceptual metrics to measure the visual impact of such artifacts. The experimental data confirmed that the subjective perception of the impairments is well-described by the measure of roughness. The subjective data of this experiment were used to obtain two perceptual metrics, named $\mathcal{R}_{1}^{*}\left(M, M^{w}\right)$ and 

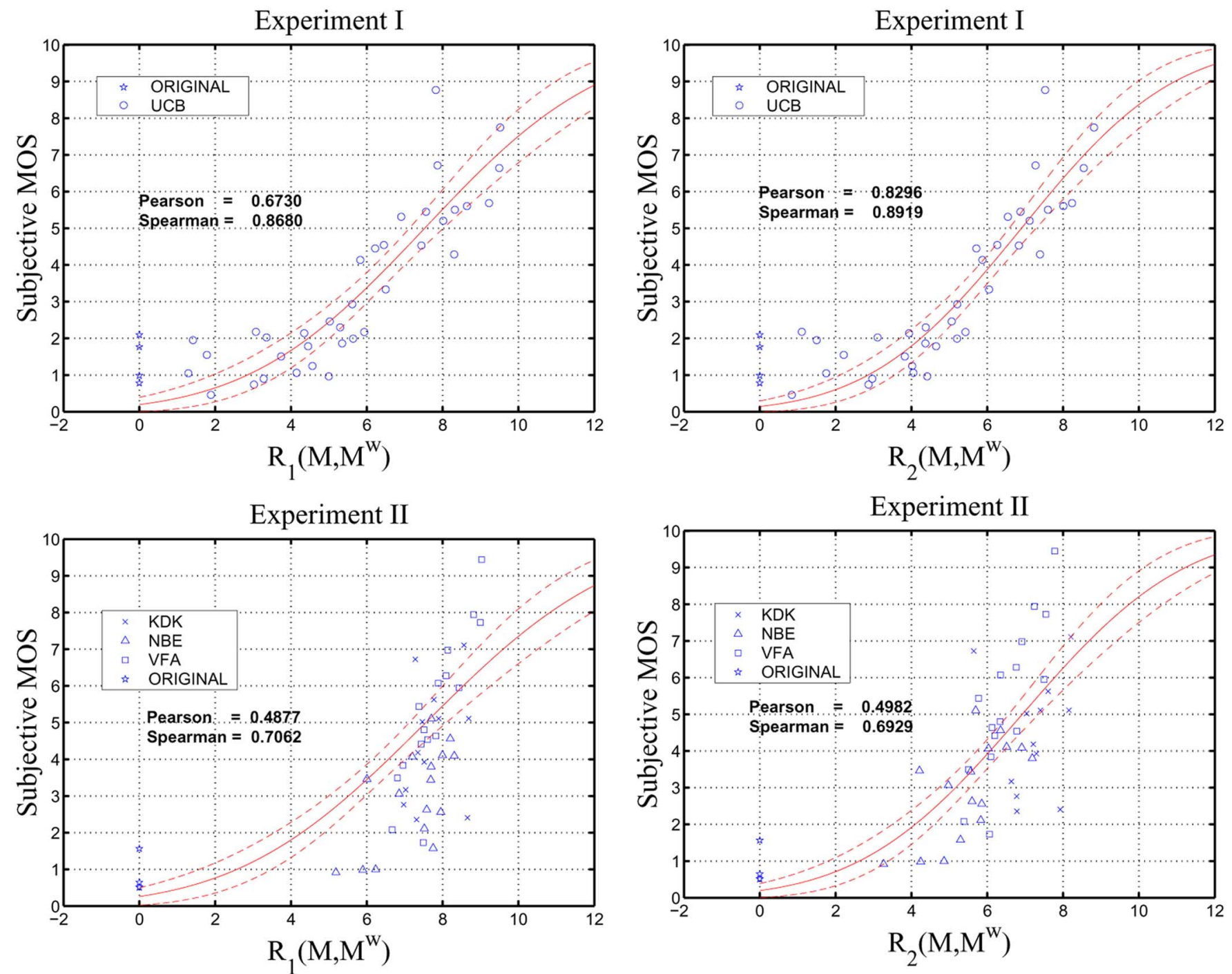

Fig. 5. Experiment I and Experiment II: Subjective $M O S$ versus objective metrics curves fits. In Experiment II, the parameters of the fitting curve are the same as Experiment I.

$\mathcal{R}_{2}^{*}\left(M, M^{w}\right)$, from the corresponding proposed objective metrics $\mathcal{R}_{1}\left(M, M^{w}\right)$ and $\mathcal{R}_{2}\left(M, M^{w}\right)$. These perceptual metrics were obtained by fitting the subjective data with the Gaussian psychometric curve in (10), leading to two perceptual metrics. The parameters of the Gaussian psychometric curve after the fitting were $(a=1.9428, b=-0.2571)$ for $\mathcal{R}_{1}\left(M, M^{w}\right)$ and $(a=$ $2.0636, b=-0.2981)$ for $\mathcal{R}_{2}\left(M, M^{w}\right)$. The smoothing-based metric provided a better fit $\left(r_{P}=0.82, r_{S}=0.89\right)$ than the multiscale $\left(r_{P}=0.67, r_{S}=0.86\right)$ as depicted in Fig. 5. On the right top of the graphs, it is possible to notice some points outside the fitting curve. Most of these outliers correspond to the Venus model. This is due to the fact that the Venus model represents a human face. Human face images are well-known in subjective experiments as a high-level factor attracting human attention, i.e., people perceive human faces differently, so the distortions on the Venus head are perceived as more visible and annoying with respect to other models.

\section{Perceptual Metrics Performance}

As discussed in the previous section, the two proposed objective metrics have been transformed into two perceptual metrics by using the data from Experiment I. In order to evaluate such metrics, Experiment II was carried out with three other watermarking algorithms: KDK, NBE, and VFA. The validation is very simple: the perceptual metrics obtained in Experiment I are used to predict the $M O S$ obtained in the second experiment and their correlation coefficients are computed. The correlation coefficients $r_{P}$ and $r_{S}$ are reported in Table I. The rows indicate which watermarking algorithms were applied to the 3-D models. The first two columns of this table report the Spearman correlation coefficients of the maximum and mean geometric errors for comparison. The third and the fourth show the values of $r_{P}$ and $r_{S}$ for $\mathcal{R}_{1}^{*}\left(M, M^{w}\right)$, while the last two columns are the $r_{P}$ and $r_{S}$ values for $\mathcal{R}_{2}^{*}\left(M, M^{w}\right)$. By referring to this table, the following considerations are in order.

- Overall, both metrics based on the Hausdorff distance (reported in the first two columns) do not correlate well with the subjective data. On the other hand, the developed metrics exhibit good correlations with the subjective data, in particular concerning Spearman's coefficient.

- The first row reports the UCB's correlation coefficients values for reference. 
- Spearman's coefficients for the NBE and VFA algorithms (second and third rows respectively) demonstrate that both metrics are able to predict impairment introduced by these two algorithms.

- The worst performance of the proposed metrics are obtained for the KDK algorithm. This can be explained by considering that the distortions produced by this algorithm are nonuniform.

- The fifth row summarizes the overall performance of the proposed metrics for the other three watermarking algorithms tested in Experiment II. The values of correlation coefficients $\left(r_{S}=0.71\right.$ for the first metric, $r_{S}=0.6929$ for the second metric) outperform the results provided by the state-of-the-art metrics $\left(r_{S}=0.3759\right.$ and $r_{S}=0.4853$ )

- The overall performance of the perceptual metrics for the watermarking algorithms that introduce uniform distortions are reported in the sixth row of the table. The values of the correlation coefficients $\left(r_{S}=0.8416\right.$ for the first metric and $r_{S}=0.8954$ for the second metric) are very good. Hence, one can claim that the developed metrics provide good prediction of the impairment caused by 3-D watermarking when artifacts are uniform.

In order to visualize the results of Experiment II, the graphs in Fig. 5 show the values of the objective metrics plotted versus the subjective $M O S$ for several watermarking algorithms. It is important to underline that the curves drawn on this figure do not represent the results of a fit as the same psychometric Gaussian curve obtained with the subjective data of the Experiment I are drawn for all the pictures. In other words, these graphs visualize the behavior of the KDK, the NBE and the VFA algorithms with respect to the developed perceptual metrics, that is represented by the solid curve (the dashed line is the confidence interval for that curve).

\section{Visual Masking and Texturing}

As stated in the introduction, our analysis neglected the masking effect due to texturing. Masking is a well-known perceptual effect of both human auditory and visual systems caused by the fact that the frequency content in certain channels suppressed the perceptibility of other frequencies in that channel. In 3-D model watermarking, this effect can be caused by the application of image texture on the models' surfaces or by the local peculiarity of the surface. For example, a surface that reproduces a particular fine pattern could mask the watermarking during visualization. The proposed metrics compensate well the visual masking effect caused by geometry since they rely on the relative roughness of the model. However, the developed metrics do not take into account visual masking caused by texture. Hence, due to the importance of textured 3-D models, we would like to further analyze some issues related to objects with texture mapping and the proposed metrics. Ferwerda et al. [15] were the first to deal with this issue. They demonstrated that texture images could make geometric artifacts of a surface less perceptible. In their work, the degree of masking of a texture is evaluated by taking into account the contrast, orientation and spatial frequency content of the final rendered image. Yixin Pan et al. [33] proposed a different approach related to the quality assessment (in terms of geometric and texture resolution) of 3-D models. Their work underlines that the perceptual contribution of image texture is, in general, more important than the model's geometry. According to their results, we can state that, in the general case, the perceptibility of the watermark on a textured model should be lower with respect to the same model rendered without textures. Consequently, our metrics can be considered as an "upper bound" of the detectability of the watermark on a textured model. In other words, if the impairment predicted by our metrics is low on a 3-D mesh, such impairment will be even lower for a 3-D model with textures.

\section{CONCLUSIONS}

In this work, a new experimental methodology for subjective quality assessment of watermarked 3-D objects has been proposed. The analysis of the data collected by two subjective experiments demonstrated that such a methodology is appropriate and provides reliable subjective data about quality evaluation of watermarked 3-D objects. Moreover, two perceptual metrics for 3-D watermarking impairments prediction have been developed by combining roughness estimation with subjective data. The performance of these metrics has been analyzed. The results of this analysis demonstrate the effectiveness of the proposed perceptual metrics with respect to the state-of-the-art geometric metrics commonly used for 3-D model comparison. More importantly, the experimental results show that the proposed metrics provide a good prediction of the human perception of the distortions introduced by 3-D watermarking. Hence, these metrics could be used in a feedback mechanism to tune the watermarking parameters of 3-D watermarking algorithms optimizing the watermark insertion. Concluding, we can state that despite the fact that the perceptual evaluation of geometric artifacts is a very difficult task due to the enormous number of influencing factors, the results obtained are encouraging. Further research can consider the evaluation of the proposed metrics under different rendering conditions and their extension to take into account the influence of local properties of the surface (e.g., curvature, protrusions) on the perception of geometric artifacts, as well as visual masking effects due to texture mapping.

\section{ACKNOWLEDGMENT}

The authors would like to thank J. M. Foley for his valuable inputs, particularly in subjective experiments design.

\section{REFERENCES}

[1] B. Rogowitz and H. Rushmeier, "Are image quality metrics adequate to evaluate the quality of geometric objects?", in Proc. SPIE: Human Vis. Electron. Imag. VI, B. E. Rogowitz and T. N. Pappas, Eds., 2001, vol. 4299, pp. 340-348.

[2] I. Cox and M. L. Miller, "A review of watermarking and the importance of perceptual modeling," in Proc. SPIE: Human Vis. Electron. Imag. II, Feb. 1997, vol. 3016, pp. 92-99.

[3] R. Wolfgang, C. I. Podilchuk, and E. J. Delp, "Perceptual watermarks for digital images and video," Proc. IEEE, vol. 87, no. 7, pp. 1108-1126, Jul. 1999. 
[4] C. I. Podilchuk and W. Zeng, "Image-adaptive watermarking using visual models," IEEE J. Select. Areas Commun., vol. 16, no. 4, pp. 525-539, Apr. 1998.

[5] R. G. V. Schyndel, A. Z. Tirkel, and C. F. Osborne, "A digital watermark," in Proc. IEEE Int. Conf. Image Processing '94, Austin, TX, Nov. 1994, vol. 2, pp. 86-90.

[6] F. Bartolini, M. Barni, V. Cappellini, and A. Piva, "Mask building for perceptually hiding frequency embedded watermarks," in Proc. 5th IEEE Int. Conf. Image Processing, ICIP'98, Chicago, IL, Oct. 1998, vol. I, pp. 450-454.

[7] Subjective Video Quality Assessment Methods for Multimedia Applications Recommendation p.910 International Telecommunication Union, Geneva, Switzerland, 1996.

[8] Methodology for Subjective Assessment of the Quality of Television Pictures Recommendation BT.500-11 Geneva, Switzerland, 2002.

[9] S. Winkler, E. Drelie Gelasca, and T. Ebrahimi, "Toward perceptual metrics for video watermark evaluation," in Proc. SPIE: Applic. Dig. Image Process., Aug. 2003, vol. 5203, pp. 371-378.

[10] N. Aspert, D. Santa-Cruz, and T. Ebrahimi, "Mesh: Measuring error between surfaces using the hausdorff distance," in Proc. IEEE Int. Conf. Multimedia and Expo 2002 (ICME), 2002, vol. I, pp. 705-708.

[11] R. S. P. Cignoni and C. Rocchini, "Metro: Measuring error on simplified surfaces," Comput. Graph. Forum, vol. 17, no. 2, pp. 167-174, 1998.

[12] P. Lindstrom and G. Turk, "Image-driven simplification," ACM Trans. Graph., vol. 19, no. 3, pp. 204-241, 2000.

[13] N. Williams, D. Luebke, J. D. Cohen, M. Kelley, and B. Schubert, "Perceptually guided simplification of lit, textured meshes," in Proc. 2003 Symp. Interactive 3D Graphics, Monterey, CA, 2003, pp. 113-121.

[14] M. R. Bolin and G. W. Meyer, "A perceptually based adaptive sampling algorithm," in Proc. 25th Annu. Conf. Computer Graphics and Interactive Techniques, 1998, pp. 299-309.

[15] J. A. Ferwerda, P. Shirley, S. N. Pattanaik, and D. P. Greenberg, "A model of visual masking for computer graphics," in Proc. 24th Conf. Computer Graphics and Interactive Techniques, 1997, pp. 143-152.

[16] R. Shacked and D. Lischinki, "Automatic lighting design using a perceptual quality metric," Comput. Graph. Forum, vol. 20, no. 3, 2001.

[17] E. B. Goldstein, Sensation and Perception, 4 ed. Washington, DC: Thompson, 1996.

[18] F. Uccheddu, M. Corsini, and M. Barni, "Wavelet-based blind watermarking of 3D models," in Proc. 2004 Multimedia and Security Workshop on Multimedia and Security, Magdeburg, Germany, 2004, pp. $143-154$.

[19] O. Benedens, "Two high capacity methods for embedding public watermarks into 3D polygonal models," in Proc. Multimedia and Security Workshop, ACM Multimedia 99, Orlando, FL, 1999, pp. 95-99.

[20] Benedens, "Watermarking of 3D polygon based models with robustness against mesh simplification," in Proc. SPIE: Security and Watermarking of Multimedia Contents, 1999, vol. 3657, pp. 329-340.

[21] S. Kanai, H. Date, and T. Kishinami, "Digital watermarking for 3D polygons using multiresolution wavelet decomposition," in Proc. 6th IFIP WG 5.2 Int. Workshop on Geometric Modeling: Fundamentals and Applications (GEO-6), Tokyo, Japan, Dec. 1998, pp. 296-307.

[22] F. Uccheddu, M. Corsini, M. Barni, and V. Cappellini, "A roughnessbased algorithm for perceptual watermarking of 3D meshes," in Proc. 10th Int. Conf. Virtual System and Multimedia, Ogaki City, Japan, Nov. 2004.

[23] J.-H Wu, S.-M Hu, J.-G Sun, and C.-L Tai, "An effective feature-preserving mesh simplification scheme based on face constriction," in Proc. 9th Pacific Conf. Computer Graphics and Applications, 2001, pp. $12-12$.

[24] M. Corsini, E. Drelie Gelasca, and T. Ebrahimi, "A multi-scale roughness metric for 3D watermarking quality assessment," in Workshop on Image Analysis for Multimedia Interactive Services 2005, Montreux, Switzerland, Apr. 2005.

[25] E. Drelie Gelasca, T. Ebrahimi, M. Corsini, and M. Barni, "Objective evaluation of the perceptual quality of 3D watermarking," in IEEE Int. Conf. Image Processing, Genoa, Italy, Sep. 2005, vol. I, pp. 241-244.

[26] G. Taubin, "A signal processing approach to fair surface design," in Proc. 22nd Annu. Conf. Computer Graphics and Interactive Techniques, 1995, pp. 351-358.

[27] L. Kobbelt, "Discrete fairing," in Proc. 7th IMA Conf. Mathematics of Surfaces '97, 1997, pp. 101-131.
[28] M. Desbrun, M. Meyer, P. Schröder, and A. H. Barr, "Implicit fairing of irregular meshes using diffusion and curvature flow," in Proc. 26th Conf. Computer Graphics and Interactive Techniques, 1999, pp. $317-324$.

[29] J. Lubin, "The use of psychophysical data and models in the analysis of display system performance," in Digital Images and Human Vision, A. B. Watson, Ed. Cambridge, MA: MIT Press, 1993.

[30] P. G. Engeldrum, Psychometric Scaling: A Tool for Imaging Systems Development. Winchester, MA: Imcotek Press, 2000.

[31] E. W. Weisstein, Correlation coefficient [Online]. Available: http://mathworld.wolfram.com/CorrelationCoefficient.html

[32] E. L. Lehmann and H. J. D'Abrera, Nonparametrics: Statistical Methods Based on Ranks. Englewood Cliffs, NJ: Prentice-Hall, 1998.

[33] Y. Pan, I. Cheng, and A. Basu, "Quality metric for approximating subjective evaluation of 3-D objects," IEEE Trans. Multimedia, vol. 7, no 2, pp. 269-279, Apr. 2005.

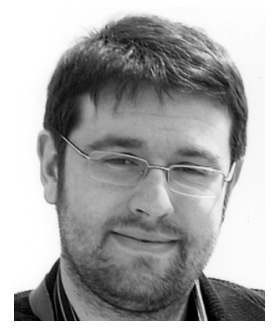

Massimiliano Corsini received the Laurea degree in information engineering in 2000, with a thesis on image mosaicing, and the Ph.D. degree in information and telecommunication engineering in 2005 , for work on 3-D watermarking of polygonal meshes and perceptual metrics for 3-D watermarking quality assessment, both from the University of Florence, Florence, Italy.

Currently, he is a Research Fellow at the Visual Computing Laboratory, Istituto di Scienza e Tecnologia dell'Informazione, National Research Council, Pisa, Italy. His research interests are in the fields of computer graphics, image processing, and computer vision, and include 3-D watermarking, perceptual metrics, appearance acquisition and modelling, and image-based relighting.

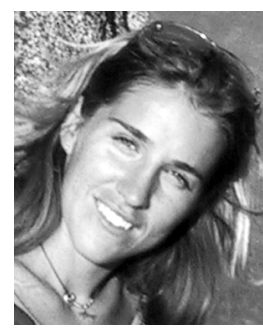

Elisa Drelie Gelasca received the Dr. Ing. degree from the Univeristy of Trieste, Treiste, Italy, in 2001 , and the Ph.D. degree from the Signal Processing Institute, Ecole Polytechnique Fédérale de Lausanne (EPFL), Lausanne, Switzerland, in 2005.

In June 2006, she joined the Vision Research Lab (VRL), University of California at Santa Barbara (UCSB), where she is currently a member of the Bioimage Informatics Group. Her research interests are in the area of image processing, with special emphasis on bioimage processing, duplicate detection, and perceptual quality assessment of video analysis and processing systems, including video segmentation and three-dimensional and video watermarking.

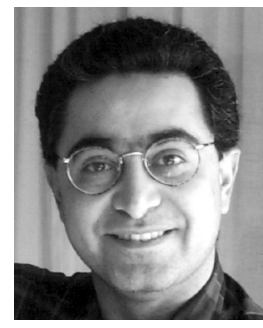

Touradj Ebrahimi (M'92) received the M.Sc. and $\mathrm{Ph} . \mathrm{D}$. degrees in electrical engineering from the Swiss Federal Institute of Technology (EPFL), Lausanne, Switzerland, in 1989 and 1992, respectively.

In 1993, he was a Research Engineer at the Corporate Research Laboratories, Sony Corporation, Tokyo, Japan, where he conducted research on advanced video compression techniques for storage applications. In 1994, he served as a Research Consultant at AT\&T Bell Laboratories, working on very-low bit-rate video coding. He is currently a Professor at EPFL, involved in research and teaching of multimedia signal processing. He is a co-founder of Genista SA, a high-tech start-up company in the field of multimedia quality metrics. In 2002, he founded Emitall SA, start-up active in the area of media security and surveillance. He has initiated more than two dozen National, European, and International cooperation projects with leading companies and research institutes around the world. $\mathrm{He}$ is also the head of the Swiss delegation to MPEG, JPEG, and SC29, and acts as the Chairman of Advisory Group on Management in SC29. His research interests include still, moving, and three-dimensional image processing and coding, visual information security (rights protection, watermarking, authentication, data integrity, steganography), new media, and human computer interfaces 
(smart vision, brain computer interface). He is the author or the co-author of more than 100 research publications and holds ten patents.

Dr. Ebrahimi has been the recipient of various distinctions, such as the IEEE and Swiss National ASE award, the SNF-PROFILE grant for advanced researchers, three ISO-Certificates for key contributions to MPEG-4 and JPEG 2000, and the Best Paper Award of the IEEE TRANSACTIONS ON CONSUMER ELECTRONICS. He became a Fellow of the International Society for Optical Engineering (SPIE) in 2003. He is or has been associate Editor with various IEEE, SPIE, and EURASIP journals, such as IEEE Signal Processing Magazine, IEEE TRANSACtions on IMAge PROCESSing, IEEE TRANSACtions ON MULTIMEDIA, the EURASIP Image Communication Journal, EURASIP Journal of Applied Signal Processing, and SPIE Optical Engineering Magazine. He is a member of the Scientific Advisory Board of various start-up and established companies in the general field of Information Technology. $\mathrm{He}$ has served as a Scientific Expert and Evaluator for Research Funding Agencies such as those of the European Commission, The Greek Ministry of Development, The Austrian National Foundation for Scientific Research, and The Portuguese Science Foundation, as well as a number of Venture Capital Companies active in the field of Information Technologies and Communication Systems. He is a member of SPIE, ACM, and IS\&T.

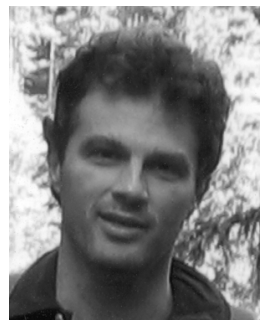

Mauro Barni (SM'06) graduated in electronic engineering in 1991 and received the Ph.D. degree in informatics and telecommunications in October 1995, both from the University of Florence, Florence, Italy.

From 1991 through 1998, he was with the Department of Electronic Engineering, University of Florence. Since September 1998, he has been with the Department of Information Engineering, University of Siena, Siena, Italy, where he is an Associate Professor. His main interests are in the field of digital image processing and computer vision. His current research activity is focused on the application of image processing techniques to copyright protection and authentication of multimedia data (digital watermarking). He is author/co-author of more than 160 papers published in international journals and conference proceedings and holds three patents in this field.

Dr. Barni is the Editor-in-Chief of the Eurasip Journal on Information Security. He is on the Editorial Board of the IEE Proceedings on Information Security, and LNCS Transactions on Data Hiding and Multimedia Security (Springer). He serves as Associate Editor of the IEEE TRANSACTIONS ON CiRcuits AND Systems For Video TeChNology, the IEEE Signal PROCESSING LetTERS, and the IEEE Signal Processing Magazine (Columns and Forum section). He is a member of the IEEE Technical Committee on Information Forensics and Security (IFS-TC). 